\title{
A study of clinical opinion and practice regarding circumcision
}

\author{
Z Farshi, K R Atkinson, R Squire
}

\begin{abstract}
Aim-To establish clinical opinion regarding appropriate indications for circumcision and to examine actual clinical practice.

Methods-A questionnaire was sent to all NHS hospital consultants in the Yorkshire region of the UK identified as having a role to play in the management of boys (under 16 years of age) requiring circumcision. Retrospective data on actual clinical practice during a three month study period were also collected via a simple proforma.

Results-Of 153 questionnaires sent, 64 were returned. Responses revealed varying opinions regarding appropriate indications for circumcision within each consultant group, and between paediatricians and surgeons. Surgeons were generally more inclined to recommend circumcision for each of the indications listed in the questionnaire. Analysis of clinical practice revealed that almost two thirds of procedures were carried out for phimosis, and nearly half of these children were under the age of 5 years.

Conclusion-There are differences in the clinical opinions of surgeons and paediatricians on what constitutes an appropriate indication for circumcision. Paediatricians' opinions are generally more in line with current evidence than those of surgeons, possibly resulting in many unnecessary circumcisions. (Arch Dis Child 2000;83:393-396)
\end{abstract}

Keywords: circumcision; clinical opinion; clinical practice; regional audit
A number of clinical reports published in British journals recommend that circumcision is indicated for established clinical reasons only and that these are present in only a small percentage of boys. ${ }^{1-4}$ However, there is evidence to suggest that the incidence of circumcision in the UK is higher than would be expected given these recommendations. ${ }^{5-9}$ It is estimated that a total of 30000 circumcisions are carried out in England each year. Department of Health statistics for 1995/96 reveal that $62 \%$ of these are performed on boys under the age of 15, with the Yorkshire region ranking sixth highest in terms of the number carried out on boys of this age. ${ }^{78}$ We aimed to establish clinical opinion on appropriate indications for circumcision and examine actual clinical practice in the Yorkshire region.

\section{Patients and methods}

To establish clinical opinion on appropriate indications for circumcision, we designed a questionnaire based on a list of indications for surgery, highlighted in a review of circumcision in childhood. ${ }^{1}$ Table 1 lists these indications. We added pain as a presenting symptom because we believe that children are commonly referred for this reason. However, very little has been written about pain as an indication for circumcision.

We sent questionnaires to all NHS hospital consultants in the Yorkshire region whom we identified as having a potential role in the management of boys requiring circumcision. These were paediatric surgeons and paediatricians, as well as general surgeons and urologists from those hospitals where there was no paediatric surgical service. We asked respondents to indicate whether they would "always", "sometimes", "rarely", or "never" consider

Table 1 Opinions regarding appropriate indications for circumcision

\begin{tabular}{|c|c|c|c|c|c|c|}
\hline \multirow[b]{2}{*}{ Indication for surgery } & \multicolumn{2}{|c|}{ Always and sometimes } & \multicolumn{2}{|c|}{ Rarely and never } & \multicolumn{2}{|c|}{ Unsure and no response } \\
\hline & Paediatricians & Surgeons & Paediatricians & Surgeons & Paediatricians & Surgeons \\
\hline \multicolumn{7}{|l|}{ Painless non-retractile foreskin } \\
\hline$<5 \mathrm{y}$ & 0 & 15 & 100 & 85 & 0 & 0 \\
\hline $5-10 y$ & 0 & 33 & 92 & 67 & 8 & 0 \\
\hline \multicolumn{7}{|l|}{ Painful non-retractile foreskin } \\
\hline$<5 \mathrm{y}$ & 24 & 56 & 48 & 44 & 28 & 0 \\
\hline $5-10 y$ & 40 & 87 & 32 & 10 & 28 & 3 \\
\hline Painful retractile foreskin & 16 & 56 & 56 & 41 & 28 & 3 \\
\hline Ballooning & 36 & 49 & 64 & 46 & 0 & 5 \\
\hline Balanitis xerotica obliterans & 40 & 90 & 0 & 5 & 60 & 5 \\
\hline \multicolumn{7}{|l|}{ Balanoposthitis } \\
\hline 1st episode & 12 & 18 & 76 & 79 & 12 & 3 \\
\hline Recurrent & 76 & 92 & 16 & 0 & 8 & 8 \\
\hline \multicolumn{7}{|l|}{ Recurrent UTI } \\
\hline Normal urinary tract & 0 & 36 & 100 & 59 & 0 & 5 \\
\hline Abnormal urinary tract & 36 & 31 & 64 & 59 & 0 & 10 \\
\hline Paraphimosis & 80 & 90 & 0 & 10 & 20 & 0 \\
\hline Cancer prevention & 4 & 8 & 68 & 84 & 28 & 8 \\
\hline
\end{tabular}

Department of Clinical

Effectiveness and

Department of

Paediatric Surgery,

St James's University

Hospital, Leeds

LS9 7TF, UK

Z Farshi

K R Atkinson

R Squire

Correspondence to: Mr Squire

email: Roly.squire@

gw.sjsuh.northy.nhs.uk

Accepted 1 June 2000

Percentages responding: paediatricians $(n=25)$, surgeons $(n=39)$. 


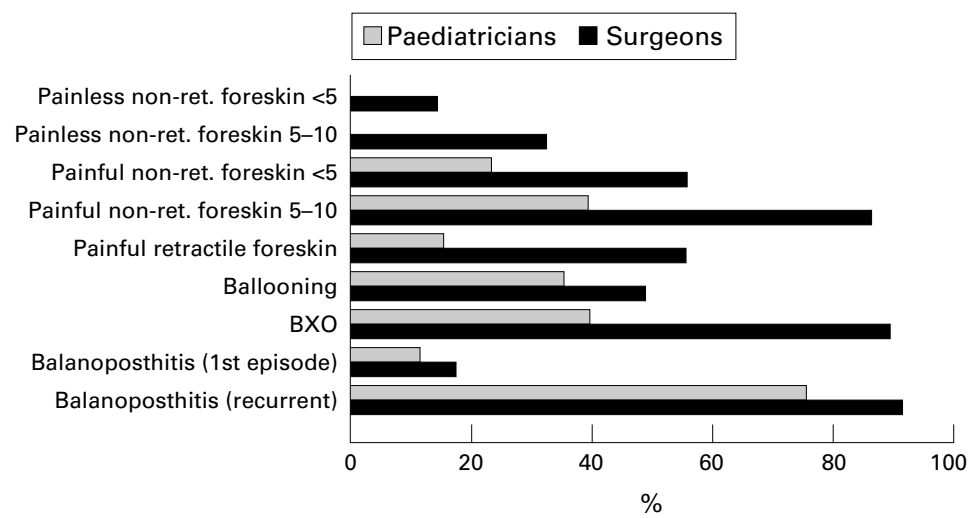

Figure 1 Indications for surgery: percentage combined responses of "always" and "sometimes" from paediatricians and surgeons.

circumcision for each of the indications listed in the questionnaire, or whether they were "unsure".

To examine actual clinical practice, we designed a proforma to obtain information about the age of patients undergoing circumcision and the documented indication for surgery. We sent a letter to surgeons working in all 14 NHS hospitals in the Yorkshire region, stating the purpose of the study and requesting permission to include their patients. Copies of the proforma were sent to the clinical audit department at each hospital where surgeons had agreed to participate. Details of patients undergoing circumcision were extracted from the central information system held at each hospital. Patient details were obtained from hospital case notes and recorded on the proforma.

Completed proformas from each hospital were returned to the lead investigator. Individual surgeons were allocated a personal identification code so they could compare their practice with that of others. This process ensured a high level of confidentiality throughout the study.

\section{Results}

We sent 153 questionnaires to 78 surgeons and 75 paediatricians. Sixty four questionnaires were returned, 39 from surgeons (response rate $50 \%$ ) and 25 from paediatricians (response rate $33 \%$ ).

Comparison of the responses, as presented in table 1 , reveals that the opinions of surgeons and paediatricians vary, with surgeons generally more inclined to recommend circumcision, most apparent for balanitis xerotica obliterans (BXO), though this may be because some paediatricians are less familiar with this diagnosis.

Table 2 Surgeons' actual clinical practice, classified by age of patient

\begin{tabular}{|c|c|c|c|c|}
\hline \multirow[b]{2}{*}{ Surgeons' recorded reason for surgery } & \multicolumn{3}{|l|}{ Age (y) } & \multirow[b]{2}{*}{ Total } \\
\hline & $<5$ & $5-10$ & $>10$ & \\
\hline Phimosis & $41(47)$ & $34(39)$ & $13(15)$ & $88(64)$ \\
\hline Balanoposthitis & $2(25)$ & $6(75)$ & - & $8(6)$ \\
\hline Painful non-retractile foreskin & - & $3(75)$ & $1(25)$ & $4(3)$ \\
\hline Balanitis xerotica obliterans & $2(14)$ & $6(43)$ & $6(43)$ & $14(10)$ \\
\hline Urinary tract infection & $1(100)$ & - & - & $1(1)$ \\
\hline Other/combination & $14(64)$ & $7(32)$ & $1(4)$ & $22(16)$ \\
\hline Total & $60(44)$ & $56(41)$ & $21(15)$ & $137(100)$ \\
\hline
\end{tabular}

Results expressed as n (\%).
However, surgeons also showed more inclination to circumcise boys for more controversial indications such as non-retractile foreskins, pain, ballooning, and balanoposthitis. Figure 1 illustrates combined responses of "always" and "sometimes" from paediatricians and surgeons, further highlighting the variation in opinion between the two consultant groups.

We observed this variation of opinion within each consultant group for most indications, the widest variations being with regard to ballooning during micturition, and urinary tract infections.

Eleven of the 14 hospitals invited to participate in the clinical study returned data, reporting 160 boys having undergone circumcision during the three month data collection period. A total of 137 boys underwent circumcision for medical reasons (the remaining 23 were carried out for religious reasons). Table 2 lists the medical indications for circumcision, classified by age groups. Overall $64 \%$ of circumcisions were carried out for "phimosis" and nearly half of these children were under 5 years of age, when pathological tightness of the foreskin almost never occurs.

\section{Discussion}

There have been few studies of this nature carried out, and we consider that our study raises important issues regarding current clinical practice. Approximately $6 \%$ of boys undergo medical circumcision in England, yet evidence from the available literature implies that medical indications actually occur in fewer than $2 \%$. $^{2}$ Our findings provide further evidence to support this discrepancy.

The findings from the questionnaire highlight differences of opinions between paediatricians and surgeons, and we are concerned that surgeons seem more inclined to recommend circumcision for non-retractability than their paediatric colleagues. We believe that in many situations evidence supports a non-operative approach. It was reported in 1949 that the normal foreskin is seldom retractable at birth, and $6 \%$ of normal boys aged 5 or over still cannot retract the foreskin. ${ }^{10}$ This was supported by data suggesting that most boys who have a non-retractable foreskin at the age of 5 will have a normally retractable prepuce at puberty, with only $1 \%$ having a pathological problem. ${ }^{11}$ As a result of these studies it is widely accepted among paediatric surgeons that unless there is a pathological process such as $\mathrm{BXO}$, the foreskin should become retractable without the need for intervention. BXO is thought to occur in approximately $1 \%$ of boys, is extremely rare under the age of 5 , and most commonly occurs as a secondary event to a prepuce that has previously been retracted. ${ }^{2}$

We identified 41 children under the age of 5 , circumcised for "phimosis". Phimosis is a term taken from a Greek word meaning "muzzled", but it is not clear whether this should include physiological non-retractibility of the prepuce, or just refer to pathological conditions. Because of this lack of clarity we did not use it in our questionnaire. However, we found it to be the most common term used as an indication for 
surgery in clinical practice. While it is extremely unlikely that the children under 5 years of age had pathological phimosis, some of the 47 children aged over 5 years who underwent circumcision for phimosis may have had BXO. It is interesting that the majority $(74 \%)$ of surgeons appear to recognise $\mathrm{BXO}$ as an important condition, yet half of the paediatricians were unsure of its significance.

We note that $49 \%$ of surgeons and $36 \%$ of paediatricians considered ballooning of the foreskin an indication for surgery. There is no evidence that ballooning is a pathological event occurring commonly in healthy but incompletely retractable foreskins, and generally does not occur when there is a pathological process such as $\mathrm{BXO}^{2}{ }^{12}$ We believe it is relevant that these issues are not fully covered in standard paediatric and surgical textbooks.

We asked how the association of pain with non-retractability influences the clinical approach. In this context, pain may refer to soreness of the prepuce, possibly because of chemical irritation caused by contact with urine, or discomfort when trying to retract a "tight" foreskin. Not surprisingly pain biases both consultant groups towards circumcision, with surgeons more inclined towards a surgical solution than the paediatricians. We are not aware of any published data that include pain when assessing the indication for surgery, yet it must be one of the most common preputial problems that leads to children seeing their general practitioner, and then being referred to a specialist.

The evidence regarding balanitis is less clear. While $3-4 \%$ of boys have one episode of balanoposthitis, less than half (probably $1 \%$ of boys overall) have recurrent episodes. ${ }^{1}$ In our study paediatricians and surgeons largely agreed that to carry out a circumcision following a single infection is unwarranted. Alternative options are available for recurrent balanitis, but surgery remains an option.

The role of circumcision in preventing urinary tract infection is controversial, but current data would suggest that while neonatal circumcision reduces the incidence of infection in childhood, the risk of renal damage is not reduced. ${ }^{13}{ }^{14}$ Urinary infection may provide a theoretical indication for circumcision, particularly in the presence of an abnormal urinary tract which has been complicated by recurrent infections. However, there is no published evidence to support this approach and routine circumcision to prevent urinary tract infection is not recommended. ${ }^{15}$ The variation in opinions which we found may reflect the level of uncertainty in this area.

There is no published evidence to support carrying out circumcisions on boys following an episode of paraphimosis, or to prevent subsequent cancer. Our study identified some clinicians who might disagree with this statement, but it is worth mentioning that paraphimosis is rare in children, and the clinical study did not identify any children in whom this indication was recorded.

We aimed to build a picture encompassing the opinions and practice of specialists in a sin- gle region of England. Difficulties encountered in getting complete data are inevitable in this context. The overall response to the questionnaire was disappointing. The results were anonymised, so it is not possible to ascertain if the $33 \%$ of paediatricians, or the $50 \%$ of surgeons who replied were a representative sample, or even if the surgeons included were the same as those actually carrying out the circumcisions in the clinical study. No attempt was made to verify the diagnoses that were quoted as an indication for circumcision, and as the majority was carried out for "phimosis" some of the data should be interpreted with caution. Despite these reservations we believe that the data reflect current attitudes of consultants to foreskin problems.

Because of a lack of available data we were unable to calculate the incidence of circumcision within the participating hospitals. Using Department of Health episode statistics we have ranked 14 regions of England in terms of the number of circumcisions performed on boys. Yorkshire region ranks sixth highest, carrying out $8 \%$ of these circumcisions nationally. Recent published evidence recommends that circumcisions should be carried out for established clinical reasons only; on that basis we suggest that up to 88 (64\%) circumcisions carried out for phimosis in the three month study period may have been unnecessary. ${ }^{17}$ If our data reflect the national picture, then movement towards more evidence based practice would result in a significant reduction in workload, cost and most importantly, unnecessary operations. Circumcisions are not always uncomplicated and there is increasing public concern that unwarranted male circumcision constitutes a form of abuse. ${ }^{16}{ }^{17}$

We have shown a widespread discrepancy between the opinions of the two consultant groups studied. In some areas this may be because the evidence is poor, so that there are grounds for controversy. However, it is likely that lack of knowledge of existing evidence has a major impact in this field. Paediatricians may perceive this to be a surgical problem and thus not their responsibility. Yet much of the data are in the paediatric domain, reflected in this study by the fact that paediatricians' opinions are more in line with current evidence. Perhaps paediatricians should bear some responsibility (where possible) for ensuring that children managed by surgical colleagues receive appropriate treatment.

The authors would like to acknowledge the contributions made from clinical audit staff in participating hospitals, consultants from clinical audit staff in participating hospitals, consultants
who participated in the study, and/or agreed for their patients to who participated in the study, and/or agreed for their patients to Clinical Audit Unit, The University of Hull, for providing fund-
Cling Clinical Audit Unit,
ing for the study.

1 Medical indications for childhood circumcision. Drug Ther Bull 1993;31(25).

2 Rickwood AMK, Walker J. Is phimosis over-diagnosed in boys and are too many circumcisions performed in consequence? Ann R Coll Surg Engl 1989;71:275-7.

3 Escala JM, Rickwood AMK. Balanitis. Br f Urol 1989;63: 196-7.

4 Williams N, Chell J, Kapila L. Why are children referred for circumcision? BMF 1993;306:28.

5 Liverpool Public Health Observatory. Male circumcision; issues. Series No. 8. Liverpool: University of Liverpool,
iverions 1996. 
6 Wyn Jones D. A review of surgical services to children in general surgery, paediatric surgery and urology in the Yorkshire re: NHS Executive, Northern \& Yorkshir Region, 1994.

7 Department of Health. Number of primary circumcision operations performed in NHS hospitals. In: Hospital Episode Statistics 1995/96. London: Department of Health, 1996.

8 Office of National Statistics, Based National Population Projections. Resident population estimates mid-year 1996. London: Stationery Office, 1999.

9 Lawrence J. Doctors campaign for end to infant circumcision. Independent Newspapers UK, 13 July 1998.

10 Gairdner D. The fate of the foreskin. A study of circumcision. BMF 1949;2:1433-7.
11 Oster J. Further fate of the foreskin. Arch Dis Child 1968;43: 200-3.

12 Rickwood AMK. Medical indications for circumcision. $\mathrm{Br} F$ Urol 1999;83:45-51.

13 Craig JC, Knight JF, Sureshkumar P, et al. Effect of circumcision on incidence of urinary tract infection in pre-school boys. F Pediatr 1996;128:23-7.

14 Guisberg CM, McCrachen GH Jr. Urinary tract infections in young children. Paediatrics 1982;69:409-12.

Poland RL. The question of routine neonatal circumcision. N Engl F Med 1990;322:1312-15.

16 William N, Lapila L. Complications of circumcision. $\mathrm{Br} \mathcal{F}$ Surg 1993;80:1231-6.

17 Warren JP, Smith PD, Dalton JD, et al. Circumcision of children. BMF 1996;312:377.

\section{TRANSATLANTIC TOPIC}

\section{Use of the new pneumococcal conjugate vaccine in the USA}

In February 2000, the Food and Drug Administration approved the new heptavalent pneumococcal conjugate vaccine (Prevnar) for use in the USA. This approval was based on a number of studies, the most prominent of which was the Northern Kaiser Permanente Vaccine trial involving 37868 children. ${ }^{1}$ In this study, children were randomised to receive either the heptavalent pneumococcal vaccine or an experimental vaccine of meningococcal $\mathrm{C}$ at $2,4,6$, and 12 to 15 months of age. Children were followed for up to 24 months after vaccination. There were three cases of vaccine serotype-specific invasive disease in the children randomised to receive the pneumococcal vaccine, and 49 cases in the children randomised to receive the meningococcal vaccine (94\% efficacy). Against bacteraemic pneumonia, the estimated efficacy was at least $85 \%$ against serotype-specific disease and there were $10 \%$ less cases of acute otitis media in the group that received the pneumococcal vaccine. More importantly, the use of tympanostomy tubes was decreased by $20 \%$ in children who had received the pneumococcal vaccine.

Based predominantly on the Kaiser data, the American Academy of Pediatrics has made the following recommendations ${ }^{2}$ :

- All children 23 months and younger should receive the vaccine at 2, 4, 6, and 15 months

- Doses of the vaccine may be administered concurrently with other childhood immunisations

- All children 23 months and younger who have not received doses of the vaccine should be given catch-up doses

- Children 24 to 59 months old who are at high risk and presumed high risk for invasive pneumococcal infection should receive the vaccine (high risk is defined as an attack rate of invasive pneumococcal disease $>150 /$ 100000 cases/year and includes children with sickle cell disease, splenic abnormalities, human immunodeficiency infection, primary immunodeficiency, and children receiving immunosuppressive treatment)

- Currently available data are inadequate to recommend use of the vaccine in children at moderate risk for invasive pneumococcal disease (moderate risk is defined as an attack rate of pneumococcal disease $>20$ cases/ 100000 per year, and includes all children 24 to 25 months old; children 36 to 59 months old who attend out of home care; and children 36 to 59 months old who are Native American or African American.

Where do these recommendations leave clinicians in the USA? Essentially, it is recommended that the current birth cohort be immunised with the new pneumococcal vaccine and that practitioners provide catch-up doses to all children less than two years old, and those between two and five years old who are at high risk of pneumococcal disease. Each vaccination costs approximately $\$ 50-80$, and thus the cost to vaccinate the 4000000 children born in the USA this year will be between $\$ 800000000$ and $\$ 1280000000$.

H BAUCHER MD US Associate Editor

1 Black S, Shienfield H, Fireman B, et al. Efficacy, safety and immunogenicity of heptavalent pneumococcal conjugate vaccine in children. Pediatric Infect Dis f 2000;19:187-95.

2 Committee on Infectious Diseases, American Academy of Pediatrics. Policy Statement: Recommendations for the prevention of pneumococcal infections, including the use of pneumococcal conjugate vaccine (Prevnar), pneumococcal polysaccharide vaccine, and antibiotic prophylaxis. Pediatrics 2000;106:362-6. [Accompanying technical report. Pediatrics 2000;106: 367-76.] 